Check for updates

Cite this: RSC Adv., 2017, 7, 31142

\title{
Water-assisted metal-free catalyzed cyclization of 2-alkynylarylketones: a facile approach to indenones $\uparrow$
}

\author{
Shuai Zhang, Xue-Ting Bai, Dan-Yun Chen, Pei Chen, Qian-Qian Zhang \\ and Yan-Bo Wang (D) *
}

Received 15th May 2017

Accepted 12th June 2017

DOI: $10.1039 / \mathrm{c} 7 \mathrm{ra05487d}$

rsc.li/rsc-advances

\begin{abstract}
A simple and directed synthetic strategy starting from 2-alkynylarylketones was developed for the construction of various indenones under metal-free and water-assisted conditions. This intramolecular cyclization reaction could well tolerate a wide range of functional groups, and the corresponding functionalized indenones were obtained in moderate to excellent yields (up to 94\%). In addition, the possible mechanism of this reaction may involve isobenzofuranium intermediates.
\end{abstract}

\section{Introduction}

Indenones, prevalent in natural products and materials science, have drawn a considerable amount of attention (Fig. 1). ${ }^{1}$ Because of the importance of indenones, various synthetic methods for substituted indenones have already been reported. The traditional synthesis of functional indenones included multistep intramolecular Friedel-Crafts acylation or used organometallic reagents. ${ }^{2,3}$ The use of transition metal species including $\mathrm{Rh},{ }^{4} \mathrm{Co},{ }^{5} \mathrm{Pd}^{6}$ or other metals ${ }^{7}$ as a catalyst has been developed by intermolecular or intramolecular cyclization reactions in recent decades. In contrast, metal-free synthetic strategies for constructing indenones have been less elucidated. Methyl trifluoromethanesulfonate (MeOTf)-mediated annulation of arylnitriles or isothiocyanates with aromatic alkynes to synthesize indenones has been demonstrated under mild conditions by Xi and coworkers. ${ }^{8}$ An efficient acid catalyzed rearrangement of a tetrahalo-7,7-dimethoxybicyclo[2.2.1] heptenyl system leading to indenones was described by Khan et al. ${ }^{9}$ Superacid-induced intramolecular cyclization of 1,3-diarylpropynones was described for the synthesis of indenones. ${ }^{10} \mathrm{An}$ efficient $\mathrm{I}_{2}$-catalyzed access to the synthesis of indenones from 2-alkynylbenzyl alcohols has been studied by Li et al. ${ }^{11}$ Benzoyl peroxide (BPO)-promoted radical cyclizations were also reported..$^{12}$ Although the above elegant metal-free methods have been made to date, the development of a simple and efficient metal-free synthetic approach to indenones is highly desirable.

Henan Engineering Laboratory of Flame-Retardant and Functional Materials, Institute of Fine Chemistry and Engineering, College of Chemistry and Chemical Engineering, Henan University, Kaifeng, 475004, China. E-mail: wangyanbokf@henu.edu.cn

$\uparrow$ Electronic supplementary information (ESI) available: X-ray crystallographic data for compound $2 n$. Copies of ${ }^{1} \mathrm{H}$ NMR and ${ }^{13} \mathrm{C}$ NMR of products. ESI-HRMS spectrum for detecting the reaction system. CCDC 1510707. For ESI and crystallographic data in CIF or other electronic format see DOI: $10.1039 / \mathrm{c} 7 \mathrm{ra} 05487 \mathrm{~d}$
2-Alkynylarylketones as an important class of substrates were widely investigated due to their convenient synthesis via the Sonogashira reaction ${ }^{13}$ and functionalized transformations to corresponding cyclic compounds..$^{14}$ Usually, the intramolecular cyclization of 2-alkynylarylketones infer to two active intermediates isobenzopyrylium I and isobenzofuranium II (Scheme 1). Owing to the aromatization of the heterocyclic ring, isobenzopyrylium ions as stable oxonium cations has been extensively studied in both nucleophilic addition reactions and cycloaddition reactions. ${ }^{15}$ However, the reactivity of isobenzofuranium intermediates has been less explored. ${ }^{16}$ Based on the above studies, great interest has been aroused to further explore isobenzofuranium intermediates involved in the reaction. Herein, we are the first time to report a simple and effective access to various indenones by water-assisted metal-free catalyzed intramolecular cyclization of 2-alkynylaryl-ketones under mild reaction conditions, and the possible path of this reaction may involve in isobenzofuranium intermediates.<smiles>COc1cc2c(cc1OC)C(c1ccc3c(c1)OCO3)=C([N+](=O)[O-])C2=O</smiles><smiles></smiles><smiles>O=C1C(c2ccc(O)cc2)=C(Br)c2cc(O)cc(O)c21</smiles><smiles>CCOC(=O)C1=C(c2ccccc2)c2cc(OCCCc3ccccc3)ccc2C1=O</smiles>

Fig. 1 Representative examples of bioactive and naturally occurring important indenones. 


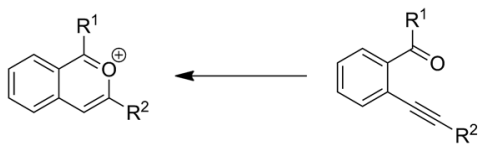

Isobenzopyrylium

Scheme 1 Intramolecular alkynylarylketones.

Table 1 Optimization of the reaction conditions for intramolecular cyclization of 2-alkynylarylketones ${ }^{a}$
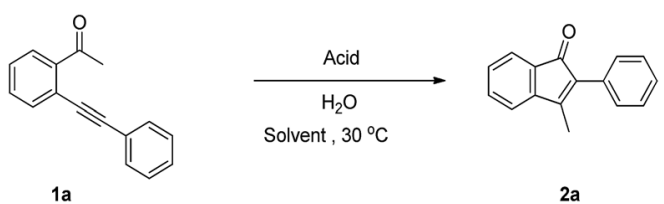

2a

\begin{tabular}{|c|c|c|c|c|}
\hline Entry & Acid (X equiv.) & $\mathrm{H}_{2} \mathrm{O}$ (X equiv.) & Solvent & Yield $^{b}(\%)$ \\
\hline 1 & $\mathrm{CH}_{3} \mathrm{COOH}(1)$ & 3 & $\mathrm{CH}_{3} \mathrm{CN}$ & - \\
\hline 2 & $\mathrm{CF}_{3} \mathrm{COOH}(1)$ & 3 & $\mathrm{CH}_{3} \mathrm{CN}$ & - \\
\hline 3 & $p-\mathrm{CH}_{3} \mathrm{C}_{6} \mathrm{H}_{4} \mathrm{SO}_{3} \mathrm{H}(1)$ & 3 & $\mathrm{CH}_{3} \mathrm{CN}$ & 18 \\
\hline 4 & $\mathrm{CH}_{3} \mathrm{SO}_{3} \mathrm{H}(1)$ & 3 & $\mathrm{CH}_{3} \mathrm{CN}$ & 33 \\
\hline 5 & $\mathrm{CF}_{3} \mathrm{SO}_{3} \mathrm{H}(1)$ & 3 & $\mathrm{CH}_{3} \mathrm{CN}$ & 40 \\
\hline 6 & $\mathrm{HCl}(1)^{c}$ & 3 & $\mathrm{CH}_{3} \mathrm{CN}$ & 85 \\
\hline 7 & $\mathrm{H}_{2} \mathrm{SO}_{4}(1)$ & 3 & $\mathrm{CH}_{3} \mathrm{CN}$ & 25 \\
\hline 8 & $\mathrm{HCl}(1)^{c}$ & 3 & DMF & - \\
\hline 9 & $\operatorname{HCl}(1)^{c}$ & 3 & DMSO & - \\
\hline 10 & $\operatorname{HCl}(1)^{c}$ & 3 & 1,4-Dioxane & 43 \\
\hline 11 & $\mathrm{HCl}(1)^{c}$ & 3 & THF & 36 \\
\hline 12 & $\operatorname{HCl}(1)^{c}$ & 3 & $\mathrm{CH}_{2} \mathrm{Cl}_{2}$ & 44 \\
\hline 13 & $\mathrm{HCl}(0.5)^{c}$ & 3 & $\mathrm{CH}_{3} \mathrm{CN}$ & 80 \\
\hline 14 & $\operatorname{HCl}(1)^{c}$ & 1 & $\mathrm{CH}_{3} \mathrm{CN}$ & 49 \\
\hline 15 & $\operatorname{HCl}(1)^{c}$ & 5 & $\mathrm{CH}_{3} \mathrm{CN}$ & 91 \\
\hline
\end{tabular}

${ }^{a}$ General reaction conditions: $\mathbf{1 a}(0.3 \mathrm{mmol})$, catalyst, solvent $(2.0 \mathrm{~mL})$, $24 \mathrm{~h}$ at $30{ }^{\circ} \mathrm{C} .{ }^{b}$ Isolated yield. ${ }^{c} \mathrm{HCl}$ in 1,4 -dioxane (4N).

\section{Results and discussion}

At the outset of our study, 2-alkynylarylketone 1a was chosen as a model substrate to optimize the reaction conditions and the experimental results are summarized in Table 1 . When the intramolecular cyclization of 2-alkynylarylketones 1a using one equivalent amount of $\mathrm{CH}_{3} \mathrm{COOH}$ or $\mathrm{CF}_{3} \mathrm{COOH}$ as catalyst and 3 equiv. $\mathrm{H}_{2} \mathrm{O}$ in $\mathrm{CH}_{3} \mathrm{CN}$ under $30{ }^{\circ} \mathrm{C}$ was attempted, no desired product $2 \mathrm{a}$ was obtained (Table 1, entries 1 and 2). Treatment of 1a with $p-\mathrm{CH}_{3} \mathrm{C}_{6} \mathrm{H}_{4} \mathrm{SO}_{3} \mathrm{H}, \mathrm{CH}_{3} \mathrm{SO}_{3} \mathrm{H}$ or $\mathrm{CF}_{3} \mathrm{SO}_{3} \mathrm{H}$ under same conditions afforded product $2 \mathrm{a}$ in $18 \%, 33 \%$, or $40 \%$ yields, respectively (Table 1, entries 3-5). To our delight, a simple and typical inorganic acid $\mathrm{HCl}$ (4N in 1,4-dioxane) was used as catalyst for the intramolecular cyclization of $\mathbf{1 a}$ to give a yield of $85 \%$ at $30{ }^{\circ} \mathrm{C}$ (Table 1 , entry 6 ). However, using another inorganic acid $\mathrm{H}_{2} \mathrm{SO}_{4}$ as catalyst led to a highly decreased yield (Table 1, entry 7). These observations clearly indicate that the properties of the acid play an important role in this transformation. Encouraged by the above results, further condition optimization of reaction solvents was conducted. In high polar
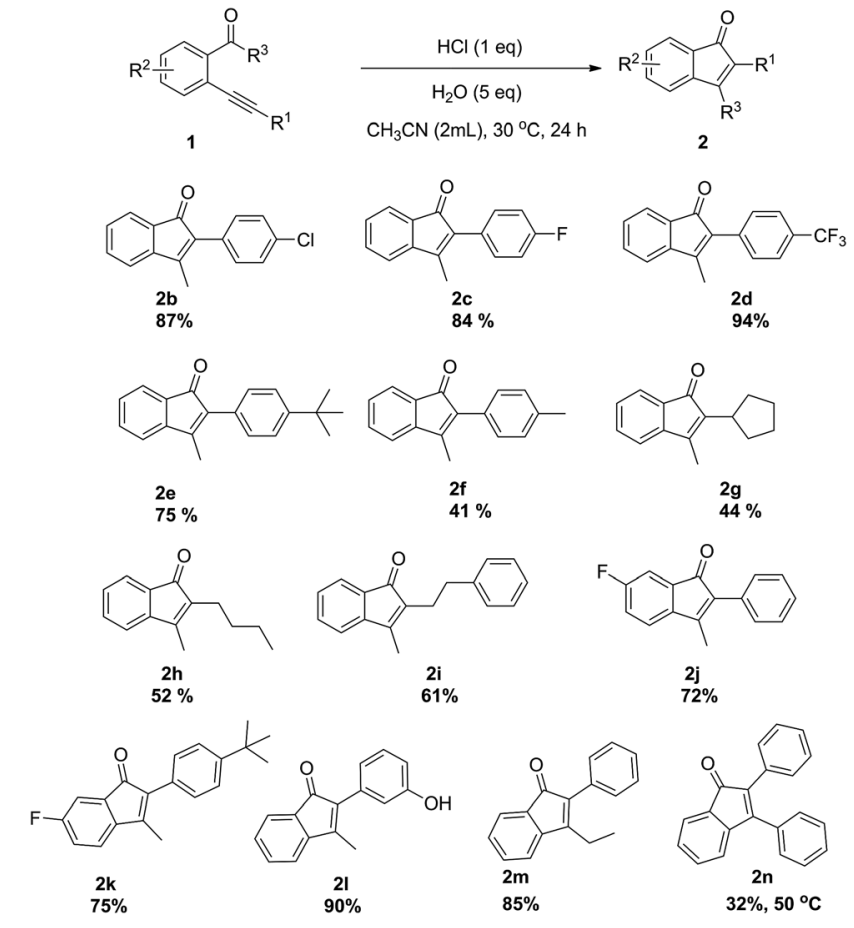

$\stackrel{2 j}{72 \%}$

Scheme 2 Intramolecular cyclization of various 2-alkynylarylketones.

solvents such as DMF or DMSO, no product 2a was obtained (Table 1, entries 8 and 9). Other solvents such as 1,4-dioxane, THF and $\mathrm{CH}_{2} \mathrm{Cl}_{2}$ afforded the product $2 \mathrm{a}$ in $43 \%, 36 \%$ or $44 \%$ yield, respectively (Table 1, entries 10-12). A slight decline in the yield of $2 \mathbf{a}$ appeared when the loading of $\mathrm{HCl}$ was decreased from 1.0 to 0.5 equiv. (Table 1 , entry 13). In addition, the loading of $\mathrm{H}_{2} \mathrm{O}$ was decreased from 3.0 to 1.0 equiv. to give an apparent decline in the yield of $2 \mathbf{a}$ (Table 1, entry 14). On the contrary, increasing the loading of $\mathrm{H}_{2} \mathrm{O}$ from 3.0 to 5.0 equiv. can accelerate the reaction and a satisfying $91 \%$ yield of $2 \mathrm{a}$ was obtained (Table 1, entry 15). Thus, the optimal reaction conditions were obtained as follows: $0.3 \mathrm{mmol} \mathrm{1a,} 1$ equiv. $\mathrm{HCl}, 5$ equiv. $\mathrm{H}_{2} \mathrm{O}$ in $2.0 \mathrm{~mL} \mathrm{CH}_{3} \mathrm{CN}$ at $30{ }^{\circ} \mathrm{C}$.

With the optimized conditions established, various substituted substrates for this intramolecular cyclization promoted by $\mathrm{HCl}$ were investigated and the results were shown in Scheme 2. It is clear that the electronic property of substituents on the phenyl ring in the $\mathrm{R}^{1}$ group exerts an obvious influence on the product yield. Substrates $\mathbf{1 b}$-d with electronwithdrawing $4-\mathrm{Cl}, 4-\mathrm{F}$ and $4-\mathrm{CF}_{3}$ groups showed higher reactivity in comparison with substrates $\mathbf{1 e - f}$ with electron-donating $4-{ }^{t} \mathrm{Bu}$ and $4-\mathrm{Me}$ groups. The corresponding products $2 \mathrm{~g}, \mathbf{2 h}$ and $2 \mathbf{i}$ were obtained in middle yields, when the alkyl-substituted alkynes $\mathbf{1 g}$, $\mathbf{1 h}$ and $\mathbf{1 i}$ were as substrates. The cyclization reactions of substrates having $4-\mathbf{F}$ (1) or $\mathbf{1 k})$ substituents on the phenyl ring in the $\mathrm{R}^{2}$ group were efficiently catalyzed to give the corresponding products $\mathbf{2} \mathbf{j}-\mathbf{k}$ in high yields. In addition, substrate having 3-OH (11) substituent on the phenyl ring in the $\mathrm{R}^{1}$ group was smoothly transformed into the corresponding product $2 \mathbf{l}$ in excellent yield. The steric effect of the $\mathrm{R}^{3}$ group on the reaction was also investigated. The reaction of substrate $\mathbf{1 m}$ 

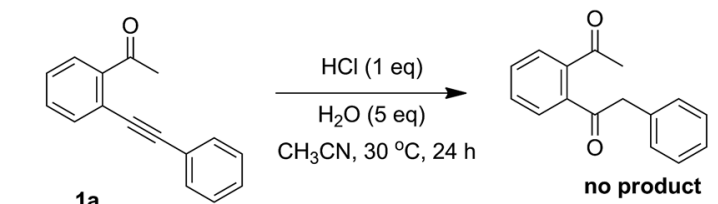

1a
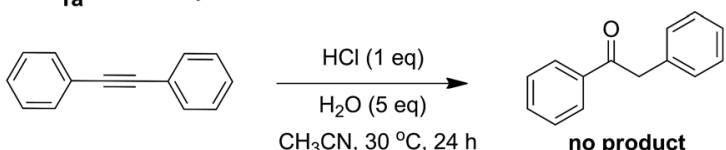

(b)
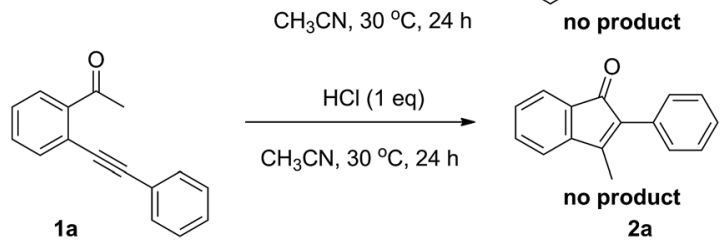

$2 a$

Scheme 3 Control experiments.

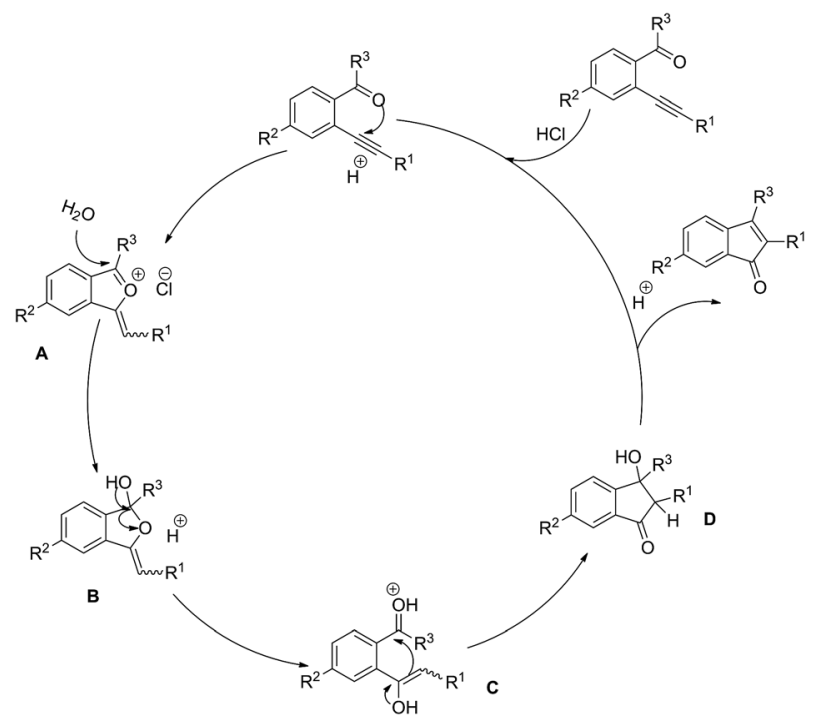

Scheme 4 Possible mechanism for intramolecular cyclization of 2alkynylarylketones.

with an ethyl group gave a good yield, whereas that of substrate 1n with phenyl group gave desired product $2 \mathrm{n}$ in only $35 \%$ yield at $50{ }^{\circ} \mathrm{C}$. The structure of $2 \mathrm{n}$ was unambiguously determined by $\mathrm{X}$-ray crystallography (see $\mathrm{ESI} \dagger$ ).

To understand the mechanistic pathway more clearly, some control experiments were carried out (Scheme 3 ). To exclude the hydration of the triple of substrates 1, 1a and 1,2-diphenylethyne failed to afford the corresponding hydration product under the standard conditions (Scheme $3 \mathrm{a}$ and b). Additionally, the experimental result showed that no product $2 \mathbf{a}$ was given under anhydrous conditions (Scheme 3c). This result indicates that the water is necessary to participate in this reaction. On the basis of the above results and previous work, ${ }^{7 f}$ a possible mechanism was proposed and shown in Scheme 4. When substrate is firstly activated by $\mathrm{HCl}$, a 5-exo-dig cyclization takes place to form isobenzofuranium intermediate $\mathbf{A}$. Then the new intermediate $\mathbf{B}$ is produced by the nucleophilic addition of $\mathrm{H}_{2} \mathrm{O}$ to $\mathbf{A}$. The carbon-oxygen bond cleavage could form enol intermediate $\mathbf{C}$ by protonation of the oxygen atom of isobenzofuran B. Subsequently, the intermediate D can be obtained via an intramolecular ring-closing reaction of the intermediate C. Finally, after elimination of $\mathrm{H}_{2} \mathrm{O}$ in the presence of $\mathrm{HCl}$, the product indenone is released together with the regenerated catalyst $\mathrm{HCl}$ for finishing a catalyst cycle. Furthermore, ESI-HRMS detection of the reaction mixture using 1a as substrate with $1 \mathrm{~h}$ was conducted to capture the information of reaction intermediates. The species of $\mathrm{m} / \mathrm{z} 221.0961$ ascribed to the intermediate $\mathbf{A}\left(\mathrm{R}^{1}=\mathrm{Ph}, \mathrm{R}^{2}=\mathrm{H}, \mathrm{R}^{3}=\mathrm{Me} ;\left[\mathrm{M}_{\mathrm{A}}\right]^{+}\right)$and the species of $m / z 239.1067$ ascribed to the intermediates $\mathbf{B}, \mathbf{C}$ and $\mathbf{D}$ $\left(\mathrm{R}^{1}=\mathrm{Ph}, \mathrm{R}^{2}=\mathrm{H}, \mathrm{R}^{3}=\mathrm{Me} ;\left[\mathrm{M}_{\mathrm{B}}+\mathrm{H}\right]^{+},\left[\mathrm{M}_{\mathrm{c}}\right]^{+}\right.$and $\left.\left[\mathrm{M}_{\mathrm{D}}+\mathrm{H}\right]^{+}\right)$were observed (see ESI Fig. S1† for details).

\section{Conclusions}

In summary, a simple and efficient $\mathrm{HCl}$ mediated water-assisted method to the construction of useful indenones by the intramolecular cyclization reaction of 2-alkynylarylketones is described under mild conditions. Various functional substrates could smoothly apply to this cyclization reaction. The reaction process may be initiated by 5-exo-dig cyclization of the carbonyl group with the alkyne triple bond, leading to isobenzofuranium intermediates.

\section{Experimental}

All chemicals and reagents were purchased from commercial suppliers without further purification unless otherwise stated. NMR spectra were recorded with tetramethylsilane as the internal standard. NMR spectrawere recorded on a Bruker Avance II 400M type $\left({ }^{1} \mathrm{H}\right.$ NMR, $\left.400 \mathrm{MHz} ;{ }^{13} \mathrm{C} \mathrm{NMR}, 100 \mathrm{MHz}\right)$ spectrometer. High resolution mass spectra (HRMS) were recorded on a Q-TOF mass spectrometry (Micromass, Wythenshawe, UK) equipped with Z-spray ionization source. Infrared spectra (IR) was measured using a Nicolet NEXUS FT-IR spectrophotometer. Substrates 2-alkynylarylketones were prepared by Sonogashira coupling reaction of corresponding 2-bromoacetophenones with alkyne according to the relate literature. ${ }^{7 f, 15 m, 16 e, 17}$

\section{Representative experimental procedure for the intramolecular cyclization of 2-alkynylarylketones}

Taking the intramolecular cyclization of 2-1-(2-(phenylethynyl)phenyl)ethanone (1a) as example: A $10 \mathrm{~mL}$ vial was charged with 1-(2-(phenylethynyl)phenyl)ethanone $1 \mathrm{a}(66.1 \mathrm{mg}, 0.30 \mathrm{mmol})$ and acetonitrile $(2 \mathrm{~mL})$, then $\mathrm{H}_{2} \mathrm{O}(27.0 \mathrm{mg}, 1.5 \mathrm{mmol})$ and $0.30 \mathrm{mmol} \mathrm{HCl}$ ( $4 \mathrm{~N}$ in 1,4-dioxane) was sequentially added into above solution. The vial was sealed and the reaction mixture was stirred at $30{ }^{\circ} \mathrm{C}$ for $24 \mathrm{~h}$. After cooling to room temperature, the resulting mixture was diluted with ethyl acetate $(10 \mathrm{~mL})$ and washed with brine $(10 \mathrm{~mL})$. The aqueous phase was extracted with ethyl acetate $(2 \times 10 \mathrm{~mL})$. The combined organic layers were dried over anhydrous $\mathrm{Na}_{2} \mathrm{SO}_{4}$, filtered and concentrated in vacuo. The crude reaction mixture was purified by column chromatography on silica gel (PE/EtOAc) to give 2a in 91\% yield. 
3-Methyl-2-phenyl-1H-inden-1-one (2a). This compound was obtained as a brown oil (91\% yield), ${ }^{1} \mathrm{H} \mathrm{NMR}\left(400 \mathrm{MHz}, \mathrm{CDCl}_{3}\right.$ ) $\delta$ (ppm): 7.46 (d, $J=6.8 \mathrm{~Hz}, 1 \mathrm{H}), 7.37-7.44(\mathrm{~m}, 5 \mathrm{H}), 7.30-7.34$ (m, 1H), 7.21-7.25 (m, 1H), $7.14(\mathrm{~d}, J=7.2 \mathrm{~Hz}, 1 \mathrm{H}), 2.29(\mathrm{~s}, 3 \mathrm{H})$; ${ }^{13} \mathrm{C}$ NMR (100 $\left.\mathrm{MHz}, \mathrm{CDCl}_{3}\right) \delta(\mathrm{ppm}):$ 196.51, 154.79, 145.93, 133.70, 133.43, 131.24, 130.44, 129.62, 128.97, 128.36, 127.76, 122.16, 119.53, 12.67; IR (film, $\mathrm{cm}^{-1}$ ): 3398, 3056, 2964, 1715, 1600, 1457, 1380, 1262, 1179, 1084, 1028, 916, 854, 804, 757, 699, 660, 597, 514; HRMS (ESI-TOF) calcd for $\mathrm{C}_{16} \mathrm{H}_{13} \mathrm{O}^{+}([\mathrm{M}+$ $\left.\mathrm{H}^{+}\right)$: 221.0961, found: 221.0961 .

2-(4-Chlorophenyl)-3-methyl-1H-inden-1-one (2b). This compound was obtained as an orange solid (87\% yield), mp 102.5-103.2; ${ }^{1} \mathrm{H}$ NMR (400 MHz, $\left.\mathrm{CDCl}_{3}\right) \delta$ (ppm): 7.49 (d, $J=$ $7.2 \mathrm{~Hz}, 1 \mathrm{H}), 7.40-7.45(\mathrm{~m}, 3 \mathrm{H}), 7.34-7.37$ (m, 2H), 7.28-7.30 (m, $1 \mathrm{H}), 7.18$ (d, $J=7.2 \mathrm{~Hz}, 1 \mathrm{H}), 2.31(\mathrm{~s}, 3 \mathrm{H}) ;{ }^{13} \mathrm{C}$ NMR $(100 \mathrm{MHz}$, $\left.\mathrm{CDCl}_{3}\right) \delta$ (ppm): 196.23, 155.19, 145.76, 133.86, 133.79, 132.35, $130.93,130.36,129.71,129.22,128.66,122.35,119.71,12.74 ;$ IR (film, $\mathrm{cm}^{-1}$ ): 3388, 2945, 2924, 1710, 1586, 1491, 1455, 1378, 1329, 1095, 1029, 1011, 860, 823, 750, 708, 514; HRMS (ESI-TOF) calcd for $\mathrm{C}_{16} \mathrm{H}_{12} \mathrm{ClO}^{+}\left([\mathrm{M}+\mathrm{H}]^{+}\right): 255.0571$, found: 255.0569 .

2-(4-Fluorophenyl)-3-methyl-1H-inden-1-one (2c). This compound was obtained as an orange solid (84\% yield), mp 115.1-117.0; ${ }^{1} \mathrm{H}$ NMR (400 MHz, $\left.\mathrm{CDCl}_{3}\right) \delta(\mathrm{ppm}): 7.48$ (d, $J=$ $7.2 \mathrm{~Hz}, 1 \mathrm{H}), 7.38-7.44$ (m, 3H), 7.25-7.29 (m, 1H), 7.11-7.18 (m, $3 \mathrm{H}), 2.31$ (s, 3H); ${ }^{13} \mathrm{C}$ NMR (100 MHz, $\left.\mathrm{CDCl}_{3}\right) \delta(\mathrm{ppm}):$ 196.48, $162.41\left(\mathrm{~d}, J_{\mathrm{C}-\mathrm{F}}=246.1 \mathrm{~Hz}\right), 154.71,145.86,133.83,132.55$, $131.39\left(\mathrm{~d}, J_{\mathrm{C}-\mathrm{F}}=8.0 \mathrm{~Hz}\right), 130.37,129.10,127.27\left(\mathrm{~d}, J_{\mathrm{C}-\mathrm{F}}=3.3\right.$ $\mathrm{Hz}$ ), 122.30, 119.61, 115.48 (d, $J_{\mathrm{C}-\mathrm{F}}=21.4 \mathrm{~Hz}$ ), 12.69; IR (film, $\mathrm{cm}^{-1}$ ): 3396, 3067, 2927, 1708, 1592, 1507, 1460, 1378, 1331, 1228, 1164, 1090, 1023, 834, 756, 713, 525; HRMS (ESI-TOF) calcd for $\mathrm{C}_{16} \mathrm{H}_{12} \mathrm{FO}^{+}\left([\mathrm{M}+\mathrm{H}]^{+}\right)$: 239.0867, found: 239.0866 .

3-Methyl-2-(4-(trifluoromethyl)phenyl)-1H-inden-1-one (2d). This compound was obtained as an orange solid (94\% yield), mp 80.6-82.5; ${ }^{1} \mathrm{H}$ NMR (400 MHz, $\left.\mathrm{CDCl}_{3}\right) \delta$ (ppm): 7.69 (d, $J=$ $8.4 \mathrm{~Hz}, 1 \mathrm{H}), 7.51-7.55(\mathrm{~m}, 3 \mathrm{H}), 7.43-7.47(\mathrm{~m}, 1 \mathrm{H}), 7.31(\mathrm{t}, J=$ $7.2 \mathrm{~Hz}, 1 \mathrm{H}), 7.21$ (d, $J=7.2 \mathrm{~Hz}, 1 \mathrm{H}), 2.35$ (s, 3H); ${ }^{13} \mathrm{C}$ NMR $(100$ $\left.\mathrm{MHz}, \mathrm{CDCl}_{3}\right) \delta$ (ppm): 195.90, 156.37, 145.55, 135.00, 133.97, $132.25,130.33,129.94,129.65\left(\mathrm{q}, J_{\mathrm{C}-\mathrm{F}}=32.2 \mathrm{~Hz}\right), 129.55,125.34$ $\left(\mathrm{q}, J_{\mathrm{C}-\mathrm{F}}=3.8 \mathrm{~Hz}\right), 124.30\left(\mathrm{q}, J_{\mathrm{C}-\mathrm{F}}=270.4 \mathrm{~Hz}\right), 122.51,119.98$, 12.79; IR (film, $\mathrm{cm}^{-1}$ ): 3401, 3069, 2924, 2852, 1713, 1615, 1458, 1409, 1381, 1327, 1167, 1117, 1069, 1015, 865, 838, 757, 713, 600, 515; HRMS (ESI-TOF) calcd for $\mathrm{C}_{17} \mathrm{H}_{12} \mathrm{~F}_{3} \mathrm{O}^{+}\left([\mathrm{M}+\mathrm{H}]^{+}\right)$: 289.0835 , found: 289.0830 .

2-(4-(tert-Butyl)phenyl)-3-methyl-1H-inden-1-one (2e). This compound was obtained as an orange solid (75\% yield), mp 98.1-99.2; ${ }^{1} \mathrm{H}$ NMR (400 MHz, $\left.\mathrm{CDCl}_{3}\right) \delta(\mathrm{ppm}): 7.45-7.49(\mathrm{~m}$, $3 \mathrm{H}), 7.36-7.43(\mathrm{~m}, 3 \mathrm{H}), 7.23-7.27(\mathrm{~m}, 1 \mathrm{H}), 7.16(\mathrm{~d}, J=7.2 \mathrm{~Hz}$, 1H), $2.33(\mathrm{~s}, 3 \mathrm{H}), 1.36(\mathrm{~s}, 9 \mathrm{H}) ;{ }^{13} \mathrm{C} \mathrm{NMR}\left(100 \mathrm{MHz}, \mathrm{CDCl}_{3}\right.$ ) $\delta$ (ppm): 196.82, 154.26, 150.72, 146.17, 133.70, 133.33, 130.56, 129.31, 128.85, 128.32, 125.39, 122.16, 119.41, 34.76, 31.41, 12.74; IR (film, $\mathrm{cm}^{-1}$ ): 3398, 3041, 2961, 2868, 1708, 1601, 1509, 1462, 1375, 1333, 1273, 1175, 1119, 1084, 1024, 912, 834, 756, 721, 676, 560; HRMS (ESI-TOF) calcd for $\mathrm{C}_{20} \mathrm{H}_{21} \mathrm{O}^{+}\left([\mathrm{M}+\mathrm{H}]^{+}\right)$: 277.1587, found: 277.1588 .

3-Methyl-2-( $\boldsymbol{p}$-tolyl)-1H-inden-1-one (2f). This compound was obtained as an reddish solid (41\% yield), mp 94.0-95.9; ${ }^{1} \mathrm{H}$ NMR $\left(400 \mathrm{MHz}, \mathrm{CDCl}_{3}\right) \delta(\mathrm{ppm}): 7.47(\mathrm{~d}, J=6.8 \mathrm{~Hz}, 1 \mathrm{H}), 7.38-7.42(\mathrm{~m}$, $1 \mathrm{H}), 7.30-7.32(\mathrm{~m}, 2 \mathrm{H}), 7.22-7.25(\mathrm{~m}, 3 \mathrm{H}), 7.14(\mathrm{~d}, J=7.2 \mathrm{~Hz}$, $1 \mathrm{H}), 2.38(\mathrm{~s}, 3 \mathrm{H}), 2.30(\mathrm{~s}, 3 \mathrm{H}) ;{ }^{13} \mathrm{C} \mathrm{NMR}\left(100 \mathrm{MHz}, \mathrm{CDCl}_{3}\right)$ $\delta$ (ppm): 196.77, 154.18, 146.15, 137.69, 133.70, 133.48, 130.55, 129.55, 129.15, 128.86, 128.32, 122.17, 119.42, 21.46, 12.70; IR (film, $\mathrm{cm}^{-1}$ ): 3395, 2963, 2921, 2856, 1706, 1612, 1593, 1511, 1457, 1380, 1332, 1262, 1081, 1032, 861, 820, 794, 756, 711, 680, 585, 518; HRMS (ESI-TOF) calcd for $\mathrm{C}_{17} \mathrm{H}_{15} \mathrm{O}^{+}\left([\mathrm{M}+\mathrm{H}]^{+}\right)$: 235.1117, found: 235.1116 .

2-Cyclopentyl-3-methyl-1H-inden-1-one (2g). This compound was obtained as a yellow solid (44\% yield), mp 81.6-82.8; ${ }^{1} \mathrm{H}$ NMR (400 MHz, $\mathrm{CDCl}_{3}$ ) $\delta(\mathrm{ppm}):$ 7.29-7.34 (m, 2H), 7.15 (dt, $J=$ $7.2,0.8 \mathrm{~Hz}, 1 \mathrm{H}), 6.99(\mathrm{~d}, J=7.2,1 \mathrm{H}), 2.81-2.90(\mathrm{~m}, 1 \mathrm{H}), 2.12(\mathrm{~s}$, $3 \mathrm{H}), 1.76-1.87$ (m, 6H), 1.60-1.65 (m, 2H); ${ }^{13} \mathrm{C}$ NMR (100 MHz, $\left.\mathrm{CDCl}_{3}\right) \delta(\mathrm{ppm}): 198.17,153.26,146.28,137.35,133.30,131.07$, 128.12, 121.41, 118.54, 35.74, 31.74, 26.39, 11.67; IR (film, $\mathrm{cm}^{-1}$ ): 3389, 2952, 2868, 1704, 1610, 1455, 1385, 1322, 1283, 1150, 1083, 1022, 952, 755, 716, 649, 546; HRMS (ESI-TOF) calcd for $\mathrm{C}_{15} \mathrm{H}_{17} \mathrm{O}^{+}\left([\mathrm{M}+\mathrm{H}]^{+}\right)$: 213.1274, found: 213.1273 .

2-Butyl-3-methyl-1H-inden-1-one (2h). This compound was obtained as a yellow solid (52\% yield), mp 60.2-62.1; ${ }^{1} \mathrm{H}$ NMR (400 MHz, $\left.\mathrm{CDCl}_{3}\right) \delta(\mathrm{ppm}): 7.30-7.36(\mathrm{~m}, 2 \mathrm{H}), 7.15(\mathrm{dt}, J=7.2$, $0.8 \mathrm{~Hz}, 1 \mathrm{H}), 7.01(\mathrm{~d}, J=7.2,1 \mathrm{H}), 2.27(\mathrm{t}, J=7.2,2 \mathrm{H}), 2.11(\mathrm{~s}, 3 \mathrm{H})$, 1.28-1.47 (m, 4H), $0.91(\mathrm{t}, J=7.2,3 \mathrm{H}) ;{ }^{13} \mathrm{C} \mathrm{NMR}(100 \mathrm{MHz}$, $\left.\mathrm{CDCl}_{3}\right) \delta(\mathrm{ppm}): 198.35,153.91,146.46,135.31,133.34,130.96$, 128.14, 121.60, 118.63, 31.33, 22.78, 22.57, 14.04, 11.59; IR (film, $\mathrm{cm}^{-1}$ ): 3395, 2957, 2930, 2858, 1707, 1609, 1457, 1384, 1287, 1159, 1103, 1083, 1016, 937, 756, 715, 632, 526; HRMS (ESI-TOF) calcd for $\mathrm{C}_{14} \mathrm{H}_{17} \mathrm{O}^{+}\left([\mathrm{M}+\mathrm{H}]^{+}\right)$: 201.1274, found: 201.1264.

3-Methyl-2-phenethyl-1H-inden-1-one (2i). This compound was obtained as a yellow solid (61\% yield), mp 63.1-64.9; ${ }^{1} \mathrm{H}$ NMR (400 MHz, $\mathrm{CDCl}_{3}$ ) $\delta(\mathrm{ppm}): 7.37$ (d, $\left.J=7.2 \mathrm{~Hz}, 1 \mathrm{H}\right), 7.28-$ $7.32(\mathrm{~m}, 1 \mathrm{H}), 7.22-7.26(\mathrm{~m}, 2 \mathrm{H}), 7.13-7.18(\mathrm{~m}, 4 \mathrm{H}), 6.94(\mathrm{~d}, J=$ $7.2 \mathrm{~Hz}, 1 \mathrm{H}), 2.75(\mathrm{t}, J=7.2 \mathrm{~Hz}, 2 \mathrm{H}), 2.56(\mathrm{t}, J=7.6 \mathrm{~Hz}, 2 \mathrm{H}), 1.79$ $(\mathrm{s}, 3 \mathrm{H}) ;{ }^{13} \mathrm{C} \mathrm{NMR}\left(100 \mathrm{MHz}, \mathrm{CDCl}_{3}\right) \delta$ (ppm): 198.14, 155.04, $146.19,141.64,133.68,133.38,130.86,128.74,128.42,128.28$, 126.02, 121.62, 118.81, 35.13, 25.19, 11.20; IR (film, $\mathrm{cm}^{-1}$ ): 3392, 3061, 3027, 2926, 2856, 1706, 1603, 1455, 1384, 1351, 1316, 1148, 1083, 1030, 957, 861, 754, 706, 632, 564; HRMS (ESI-TOF) calcd for $\mathrm{C}_{18} \mathrm{H}_{17} \mathrm{O}\left([\mathrm{M}+\mathrm{H}]^{+}\right)$: 249.1274, found: 249.1274 .

5-Fluoro-3-methyl-2-phenyl-1H-inden-1-one (2j). This compound was obtained as a reddish brown solid (72\% yield), mp 93.4-95.2; ${ }^{1} \mathrm{H}$ NMR (400 MHz, $\left.\mathrm{CDCl}_{3}\right) \delta(\mathrm{ppm}): 7.43-7.46(\mathrm{~m}$, 2H), 7.38-7.41 (m, 2H), 7.21 (dd, $J=7.2,2.4 \mathrm{~Hz}, 1 \mathrm{H}), 7.04-7.13$ $(\mathrm{m}, 2 \mathrm{H}), 2.32(\mathrm{~s}, 3 \mathrm{H}) ;{ }^{13} \mathrm{C}$ NMR $\left(100 \mathrm{MHz} \mathrm{CDCl}_{3}\right) \delta(\mathrm{ppm})$ : $194.85,163.81\left(\mathrm{~d}, J_{\mathrm{C}-\mathrm{F}}=248.6 \mathrm{~Hz}\right), 154.96\left(\mathrm{~d}, J_{\mathrm{C}-\mathrm{F}}=2.0 \mathrm{~Hz}\right)$, $141.37\left(\mathrm{~d}, J_{\mathrm{C}-\mathrm{F}}=3.2 \mathrm{~Hz}\right), 133.94\left(\mathrm{~d}, J_{\mathrm{C}-\mathrm{F}}=4.6 \mathrm{~Hz}\right), 132.93\left(\mathrm{~d}, J_{\mathrm{C}-\mathrm{F}}\right.$ $=7.1 \mathrm{~Hz}), 131.02,129.54,128.46,127.93,120.64\left(\mathrm{~d}, J_{\mathrm{C}-\mathrm{F}}=7.8\right.$ $\mathrm{Hz}), 118.86\left(\mathrm{~d}, J_{\mathrm{C}-\mathrm{F}}=22.9 \mathrm{~Hz}\right), 110.01\left(\mathrm{~d}, J_{\mathrm{C}-\mathrm{F}}=24.7 \mathrm{~Hz}\right), 12.87$; IR (film, $\mathrm{cm}^{-1}$ ): 3399, 3095, 3061, 2963, 2925, 2853, 1707, 1619, 1477, 1442, 1375, 1311, 1262, 1222, 1098, 1027, 892, 841, 796, 737, 701, 577, 522; HRMS (ESI-TOF) calcd for $\mathrm{C}_{16} \mathrm{H}_{12} \mathrm{FO}^{+}([\mathrm{M}+$ $\left.\mathrm{H}^{+}\right)$: 239.0867 , found: 239.0867 .

2-(4-(tert-Butyl)phenyl)-5-fluoro-3-methyl-1 $\mathrm{H}$-inden-1-one (2k). This compound was obtained as a reddish brown solid (75\% yield), mp 106.1-108.0; ${ }^{1} \mathrm{H}$ NMR (400 $\mathrm{MHz}, \mathrm{CDCl}_{3}$ ) $\delta$ (ppm): 7.46 (d, $J=8.4 \mathrm{~Hz}, 2 \mathrm{H}), 7.35$ (d, $J=8.4 \mathrm{~Hz}, 2 \mathrm{H}), 7.20$ 
(dd, $J=7.2,1 \mathrm{~Hz}, 1 \mathrm{H}), 7.03-7.13(\mathrm{~m}, 2 \mathrm{H}), 2.32(\mathrm{~s}, 3 \mathrm{H}), 1.35$ (s, $9 \mathrm{H}) ;{ }^{13} \mathrm{C} \mathrm{NMR}\left(100 \mathrm{MHz}, \mathrm{CDCl}_{3}\right) \delta(\mathrm{ppm}): 195.15,163.76\left(\mathrm{~d}, J_{\mathrm{C}-\mathrm{F}}\right.$ $=248.3 \mathrm{~Hz}), 154.44\left(\mathrm{~d}, J_{\mathrm{C}-\mathrm{F}}=2.1 \mathrm{~Hz}\right), 150.92,141.58\left(\mathrm{~d}, J_{\mathrm{C}-\mathrm{F}}=\right.$ $3.2 \mathrm{~Hz}), 133.82\left(\mathrm{~d}, J_{\mathrm{C}-\mathrm{F}}=4.6 \mathrm{~Hz}\right), 133.01\left(\mathrm{~d}, J_{\mathrm{C}-\mathrm{F}}=7.1 \mathrm{~Hz}\right)$, $129.21,128.08,125.47,120.48\left(\mathrm{~d}, J_{\mathrm{C}-\mathrm{F}}=7.8 \mathrm{~Hz}\right), 118.82\left(\mathrm{~d}, J_{\mathrm{C}-\mathrm{F}}=\right.$ $22.8 \mathrm{~Hz}), 111.00\left(\mathrm{~d}, J_{\mathrm{C}-\mathrm{F}}=24.6 \mathrm{~Hz}\right.$ ), 34.81, 31.42, 12.93; IR (film, $\mathrm{cm}^{-1}$ ): 3406, 3053, 2963, 2905, 2869, 1713, 1620, 1507, 1474, 1438, 1377, 1331, 1266, 1224, 1204, 1113, 1093, 1028, 1011, 887, 838, 787, 762, 734, 561; HRMS (ESI-TOF) calcd for $\mathrm{C}_{20} \mathrm{H}_{20} \mathrm{FO}^{+}$ $\left([\mathrm{M}+\mathrm{H}]^{+}\right): 295.1493$, found: 295.1493 .

2-(3-Hydroxyphenyl)-3-methyl-1H-inden-1-one (2l). This compound was obtained as a red solid (90\% yield), mp 128.5129.9; ${ }^{1} \mathrm{H}$ NMR (400 MHz, $d^{6}$-DMSO) $\delta$ (ppm): 9.52 (s, 1H), 7.53 $(\mathrm{td}, J=7.2,0.8 \mathrm{~Hz}, 1 \mathrm{H}), 7.42(\mathrm{~d}, J=7.2 \mathrm{~Hz}, 1 \mathrm{H}), 7.32-7.37(\mathrm{~m}$, $2 \mathrm{H}), 7.25(\mathrm{t}, J=8.0 \mathrm{~Hz}, 1 \mathrm{H}), 6.82-6.84(\mathrm{~m}, 2 \mathrm{H}), 6.76-6.79(\mathrm{~m}$, $1 \mathrm{H}), 2.31$ (s, 3H); ${ }^{13} \mathrm{C}$ NMR (100 MHz, $d^{6}$-DMSO) $\delta(\mathrm{ppm}): 195.79$, 157.11 , 155.51, 145.29, 134.18, 132.26, 131.99, 129.60, 129.21, $129.20,121.65,120.30,120.20,116.25,114.77,12.58$; IR $(\mathrm{KBr}$, $\left.\mathrm{cm}^{-1}\right): 3278,3068,2923,2853,1692,1596,1508,1440,1381$, 1345, 1314, 1285, 1257, 1233, 1167, 1089, 1035, 1000, 917, 875, 841, 812, 778, 758, 704, 668; HRMS (ESI-TOF) calcd for $\mathrm{C}_{16} \mathrm{H}_{13} \mathrm{O}_{2}^{+}\left([\mathrm{M}+\mathrm{H}]^{+}\right): 237.0910$, found: 237.0910 ; anal. calcd for $\mathrm{C}_{16} \mathrm{H}_{12} \mathrm{O}_{2}$ (\%); C, 81.34; H, 5.12. Found: C, 81.05; H, 5.07.

3-Ethyl-2-phenyl-1H-inden-1-one (2m). This compound was obtained as a yellow solid (85\% yield), mp 186.6-188.5; ${ }^{1} \mathrm{H}$ NMR $\left(400 \mathrm{MHz}, \mathrm{CDCl}_{3}\right) \delta(\mathrm{ppm}): 7.51(\mathrm{~d}, J=7.2 \mathrm{~Hz}, 1 \mathrm{H}), 7.33-7.46(\mathrm{~m}$, $6 \mathrm{H}), 7.24-7.28(\mathrm{~m}, 1 \mathrm{H}), 7.20(\mathrm{~d}, J=7.6 \mathrm{~Hz}, 1 \mathrm{H}), 2.74(\mathrm{q}, J=$ $7.6 \mathrm{~Hz}, 2 \mathrm{H}), 1.34$ (t, $J=7.6 \mathrm{~Hz}, 3 \mathrm{H}) ;{ }^{13} \mathrm{C} \mathrm{NMR}\left(100 \mathrm{MHz}, \mathrm{CDCl}_{3}\right.$ ) $\delta$ (ppm): 196.94, 160.05, 144.99, 133.69, 132.94, 131.37, 130.90, 129.45, 128.87, 128.44, 127.88, 122.50, 119.98, 20.12, 12.93; IR (film, $\mathrm{cm}^{-1}$ ): 3397, 3057, 2973, 2935, 2876, 1712, 1601, 1492, 1459, 1345, 1298, 1173, 1087, 1051, 916, 853, 837, 755, 723, 699, $665,640,590,512$; HRMS (ESI-TOF) calcd for $\mathrm{C}_{17} \mathrm{H}_{15} \mathrm{O}^{+}([\mathrm{M}+$ $\mathrm{H}^{+}$): 235.1117 , found: 235.1121 .

2,3-Diphenyl-1H-inden-1-one (2n). This compound was obtained as a red solid (32\% yield), mp 151.8-153.4; ${ }^{1} \mathrm{H}$ NMR (400 $\left.\mathrm{MHz}, \mathrm{CDCl}_{3}\right) \delta(\mathrm{ppm}): 7.59(\mathrm{~d}, J=7.2 \mathrm{~Hz}, 1 \mathrm{H}), 7.35-7.43(\mathrm{~m}, 6 \mathrm{H})$, $7.25-7.31(\mathrm{~m}, 6 \mathrm{H}), 7.15(\mathrm{~d}, J=7.2 \mathrm{~Hz}, 1 \mathrm{H}) .{ }^{13} \mathrm{C}$ NMR $(100 \mathrm{MHz}$, $\left.\mathrm{CDCl}_{3}\right) \delta(\mathrm{ppm}): 196.64,155.46,145.35,133.57,132.83,132.52$, $130.87,130.85$, 130.10, 129.42, 129.09, 128.91, 128.63, 128.20, 127.87, 123.11, 121.39; IR (film, $\mathrm{cm}^{-1}$ ): 3384, 3065, 2923, 2854, 1701, 1601, 1486, 1450, 1348, 1281, 1180, 1076, 1024, 922, 843, $755,699,585,518$; HRMS (ESI-TOF) calcd for $\mathrm{C}_{21} \mathrm{H}_{15} \mathrm{O}([\mathrm{M}+$ $\left.\mathrm{H}]^{+}\right)$: 283.1117, found: 283.1119; anal. calcd for $\mathrm{C}_{21} \mathrm{H}_{14} \mathrm{O}(\%)$; , 89.34; H, 5.00. Found: C, 89.09; H, 5.06.

\section{Acknowledgements}

This work was financially supported by the National Natural Science Foundation of China (No. U1504205) and the Key Research Project of Education Department of Henan Province (No. 17A150002).

\section{Notes and references}

1 (a) G. M. Anstead, S. R. Wilson and J. A. Katzenellenbogen, J. Med. Chem., 1989, 32, 2163; (b) J. H. Ahn, M. S. Shin,
S. H. Jung, S. K. Kang, K. R. Kim, S. D. Rhee, W. H. Jung, S. D. Yang, S. J. Kim, J. R. Woo, J. H. Lee, H. G. Cheon and S. S. Kim, J. Med. Chem., 2006, 49, 4781; (c) A. Morrell, M. Placzek, S. Parmley, B. Grella, S. Antony, Y. Pommier and M. Cushman, J. Med. Chem., 2007, 50, 4388; (d) J. L. Jeffrey and R. Sarpong, Org. Lett., 2009, 11, 5450; (e) X. Chen, J. Jin, N. Wang, P. Lu and Y. Wang, Eur. J. Org. Chem., 2012, 2012, 824.

2 (a) J. F. Feeman and E. D. Amstutz, J. Am. Chem. Soc., 1950, 72, 1522; (b) M. B. Floyd and G. R. J. Allen, J. Org. Chem., 1970, 35, 2647; (c) H. Martens and G. Hoornaert, Synth. Commun., 1972, 2, 147; (d) G. Jammaer, H. Martens and G. Hoornaert, Tetrahedron, 1975, 31, 2293; (e) M. Rostami, A. R. Khosropour, V. Mirkhani, M. Moghadam, S. Tangestaninejad and I. Mohammadpoor-Baltork, Tetrahedron Lett., 2011, 52, 7149.

3 (a) R. L. Frank, H. Eklund, J. W. Richter, C. R. Vanneman and A. N. Wennerberg, J. Am. Chem. Soc., 1944, 66, 1; (b) C. Manning, M. R. McClory and J. J. McCullough, J. Org. Chem., 1981, 46, 919; (c) K. Katsumoto, C. Kitamura and T. Kawase, Eur. J. Org. Chem., 2011, 2011, 4885.

4 Selected examples: (a) K. Kokubo, K. Matsumasa, M. Miura and M. Nomura, J. Org. Chem., 1996, 61, 6941; (b) T. Fukuyama, N. Chatani, F. Kakiushi and S. Murai, J. Org. Chem., 1997, 62, 5647; (c) T. Miura and M. Murakami, Org. Lett., 2005, 7, 3339; (d) Y. Harada, J. Nakanishi, H. Fujihara, M. Tobisu, Y. Fukumoto and N. Chatani, J. Am. Chem. Soc., 2007, 129, 5766; (e) T. Morimoto, K. Yamasaki, A. Hirano, K. Tsutsumi, N. Kagawa, K. Kakiuchi, Y. Harada, Y. Fukumoto, N. Chatani and T. Nishioka, Org. Lett., 2009, 11, 1777; $(f)$ B.-J. Li, H.-Y. Wang, Q.-L. Zhu and Z.-J. Shi, Angew. Chem., 2012, 124, 4014; Angew. Chem. Int. Ed., 2012, 51, 3948; (g) S. Chen, J. Yu, Y. Jiang, F. Chen and J. Cheng, Org. Lett., 2013, 15, 4754; (h) Z. Qi, M. Wang and X. Li, Org. Lett., 2013, 15, 5440; (i) X. Yu, Y. Duan, W. Guo, T. Wang, Q. Xie, S. Wu, C. Jiang, Z. Fan, J. Wang and G. Liu, Organometallics, 2017, 36, 1027.

5 Selected examples: (a) D. H. Kim, S. U. Son and Y. K. Chung, Org. Lett., 2003, 5, 3151; (b) W. Yu, W. Zhang, Z. Liu and Y. Zhang, Chem. Commun., 2016, 52, 6837; (c) M. Ueda, T. Ueno, Y. Suyama and I. Ryu, Chem. Commun., 2016, 52, 13237; (d) L. Kong, X. Yang, X. Zhou, S. Yu and X. Li, Org. Chem. Front., 2016, 3, 813.

6 Selected examples: (a) R. C. Larock and M. J. Doty, J. Org. Chem., 1993, 58, 4579; (b) J. Vicente, J.-A. Abad and J. GilRubio, Organometallics, 1996, 15, 3509; (c) J. Vicente, J.-A. Abad, B. López-Peláez and E. Martínez-Viviente, Organometallics, 2002, 21, 58; (d) A. A. Pletnev, Q. Tian and R. C. Larock, J. Org. Chem., 2002, 67, 9276; (e) H. Tsukamoto and Y. Kondo, Org. Lett., 2007, 9, 4227; (f) X. Chen, Q. He, Y. Xie and C. Yang, Org. Biomol. Chem., 2013, 11, 2582; $(g)$ A. N. Butkevich, B. Ranieri, L. Meerpoel, I. Stansfield, P. Angibaud, A. Corbua and J. Cossy, Org. Biomol. Chem., 2014, 12, 728; (h) B. Suchand and G. Satyanarayana, J. Org. Chem., 2017, 82, 372. 
7 Selected examples: (a) T. Fukuyama, N. Chatani, F. Kakiuchi and S. Murai, J. Org. Chem., 1997, 62, 5647; (b) Y. Kuninobu, T. Matsuki and K. Takai, Org. Lett., 2010, 12, 2948; (c) X.-Q. Pan, J.-P. Zou, G.-L. Zhang and W. Zhang, Chem. Commun., 2010, 46, 1721; (d) J. Zhou, G.-L. Zhang, J.-P. Zou and W. Zhang, Eur. J. Org. Chem., 2011, 2011, 3412; (e) J. Zhang, D. Wu, X. Chen, Y. Liu and Z. Xu, J. Org. Chem., 2014, 79, 4799; (f) M. E. Domaradzki, Y. Long, Z. She, X. Liu, G. Zhang and Y. Chen, J. Org. Chem., 2015, 80, 11360; $(g)$ D. H. Dethe and G. M. Murhade, Chem. Commun., 2015, 51, 10891.

8 (a) X. Yan, S. Zou, P. Zhao and C. Xi, Chem. Commun., 2014, 50, 2775; (b) P. Zhao, Y. Liu and C. Xi, Org. Lett., 2015, 17, 4388.

9 K. R. Babu and F. A. Khan, Org. Biomol. Chem., 2015, 13, 299. 10 (a) A. V. Vasilyev, S. Walspurger, P. Pale and J. Sommer, Tetrahedron Lett., 2004, 45, 3379; (b) A. V. Vasilyev, S. Walspurger, M. Haouas, J. Sommer, P. Pale and A. P. Rudenkoa, Org. Biomol. Chem., 2004, 2, 3483; (c) A. V. Vasil'ev, S. Walspurger, P. Pale, J. Sommer, M. Haouas and A. P. Rudenko, Russ. J. Org. Chem., 2004, 40, 1769.

11 C. Wang, J. Yang, X. Cheng, E. Li and Y. Li, Tetrahedron Lett., 2012, 53, 4402.

12 (a) C. Pan, B. Huang, W. Hu, X. Feng and J.-T. Yu, J. Org. Chem., 2016, 81, 2087; (b) X.-S. Zhang, J.-Y. Jiao, X.-H. Zhang, B.-L. Hu and X.-G. Zhang, J. Org. Chem., 2016, 81, 5710.

13 (a) E.-I. Negishi and L. Anastasia, Chem. Rev., 2003, 103, 1979; (b) R. Chinchilla and C. Nájera, Chem. Rev., 2007, 107, 874 .

14 (a) N. Chernyak, S. I. Gorelsky and V. Gevorgyan, Angew. Chem., 2011, 123, 2390; Angew. Chem. Int. Ed., 2011, 50, 2342; (b) Z.-Q. Wang, W.-W. Zhang, L.-B. Gong, R.-Y. Tang, X.-H. Yang, Y. Liu and J.-H. Li, Angew. Chem., 2011, 123, 2390; Angew. Chem. Int. Ed., 2011, 50, 8968; (c) D. Zheng, S. Li and J. Wu, Org. Lett., 2012, 14, 2655; (d) S.-Y. Yu, H. Zhang, Y. Gao, L. Mo, S. Wang and Z.-J. Yao, J. Am. Chem. Soc., 2013, 135, 11402; (e) C. Dong, Z. Liao, X. Xu and H. Zhou, J. Heterocycl. Chem., 2014, 51, 1282.
15 (a) N. Asao, T. Nogami, S. Lee and Y. Yamamoto, J. Am. Chem. Soc., 2003, 125, 10921; (b) N. Asao, T. Kasahara and Y. Yamamoto, Angew. Chem., 2003, 115, 3628; Angew. Chem. Int. Ed., 2003, 42, 3504; (c) J.-L. Zhu, A.-R. Germain and J. A. Porco Jr, Angew. Chem., 2004, 116, 1259; Angew. Chem. Int. Ed., 2004, 43, 1239; (d) N. Asao, K. Sato and Y. Yamamoto, J. Org. Chem., 2005, 70, 3682; (e) J.-L. Zhu, N. P. Grigoriadis, J.-P. Lee and J. A. Porco Jr, J. Am. Chem. Soc., 2005, 127, 9342; (f) H. Kusama, H. Funami, M. Shido, Y. Hara, J. Takaya and N. Iwasawa, J. Am. Chem. Soc., 2005, 127, 2709; (g) S. Shin, A.-K. Gupta, C.-Y. Rhim and C.-H. Oh, Chem. Commun., 2005, 41, 4429; $(h)$ D. Yue, N. D. Cá and R. C. Larock, J. Org. Chem., 2006, 71, 3381; (i) A.-B. Beeler, S. Su, C.-A. Singleton and J. A. Porco Jr, J. Am. Chem. Soc., 2007, 129, 1413; (j) Y.-C. Hsu, C.-M. Ting and R.-S. Liu, J. Am. Chem. Soc., 2009, 131, 2090; (k) L.-P. Liu and G. B. Hammond, Org. Lett., 2010, 12, 4640; (l) M. Terada, F. Li and Y. Toda, Angew. Chem., 2014, 126, 239; Angew. Chem. Int. Ed., 2014, 53, 235; (m) B. Guo, L. Zheng, L. Yang and R. Hua, J. Org. Chem., 2014, 79, 4352; (n) S. Zhu, H. Huang, Z. Zhang, T. Ma and H. Jiang, J. Org. Chem., 2014, 79, 6113; (o) Q. Xu, P. Gu, F. Wang and M. Shi, Org. Chem. Front., 2015, 2, 1475; $(p)$ T. Miao, Z.-Y. Tian, Y.-M. He, F. Chen, Y. Chen, Z.-X. Yu and Q.-H. Fan, Angew. Chem., 2017, 129, 4199; Angew. Chem. Int. Ed., 2017, 56, 4135; (q) J. Sun, J.-K. Qiu, Y.-N. Wu, W.-J. Hao, C. Guo, G. Li, S.-J. Tu and B. Jiang, Org. Lett., 2017, 19, 754.

16 (a) D. Jiang and J. W. Herndon, Org. Lett., 2000, 2, 1267; (b) Y. Luo, J. W. Herndon and F. Cervantes-Lee, J. Am. Chem. Soc., 2003, 125, 12720; (c) T. Godet, C. Vaxelaire, C. Michel, A. Milet and P. Belmont, Chem.-Eur. J., 2007, 13, 5632; (d) K. Sekine, A. Takayanagi, S. Kikuchi and T. Yamada, Chem. Commun., 2013, 49, 11320; (e) W.-Z. Zhang, L.-L. Shi, C. Liu, X.-T. Yang, Y.-B. Wang, Y. Luo and X.-B. Lu, Org. Chem. Front., 2014, 1, 275.

17 (a) M. Dell'Acqua, G. Abbiati, A. Arcadi and E. Rossi, Org. Biomol. Chem., 2011, 9, 7836; (b) S. Manojveer and R. Balamurugan, Org. Lett., 2014, 16, 1712. 